\title{
Towards an ICT-TPCK based design: Hybrid Solution for the development of soft skills in Higher Education
}

\section{Progettare in prospettiva ICT-TPCK: Hybrid Solution per lo sviluppo di soft skill in Higher Education}

\author{
Marina De Rossi ${ }^{1}$
}

\begin{abstract}
The implementation of the ICT-TPCK integrated design procedures (Technological, Pedagogical, Content Knowledge) can foster an effective teachers' planning thinking. The aim is to consider all the elements as essential to develop an effective integration of ICT in teaching in HIS (Hybrid Instruction Solution) learning environments. A mixed methods study was carried out in 2017-18 involving 42 volunteer teachers. The goal was to design and to implement some onsite/online hybrid solutions to enhance specific soft skills in the field of curricular disciplinary teaching. A structured questionnaire was built, administered in and out, and could lead to interesting reflections on some issues that are still open in the Higher Education area: the quality and objectives of teacher training; the effective overcoming of the frontal lesson; the relationship between technological affordances and methodological approaches.
\end{abstract}

Keywords: ICT-TPCK, Higher Education, Instructional design, Active Learning, Soft Skill.

\section{Introduzione}

Il significato del termine "hybrid solution" (hybrid instruction solution HIS) (Kaleta, Skibba \& Joosten, 2007; Dziuban, Moskal, Kramer \& Thompson, 2013), spesso usato in modo intercambiabile con "blended solution", è ampio e comprensivo di diversificati format didattici sviluppati attraverso l'allestimento di ambienti d'apprendimento misti che richiedono mirata progettazione (Shea, 2007).

Infatti, andrebbero considerate "l'integrazione non solo di elementi legati alla spazialità, reale o virtuale che sia, ma anche delle modalità comunicative (sincrone e asincrone), delle strategie didattiche da adottare nei diversi momenti e nei diversi spazi in cui si sviluppa il processo di insegnamento-apprendimen-

\footnotetext{
${ }^{1}$ Marina de Rossi è professore associato presso l'Università degli Studi di Padova.
} 
to, dei diversi strumenti tecnologici e delle risorse per l'apprendimento da utilizzare a supporto dello studio individuale e/o collaborativo" (Trentin \& Bocconi, 2015, p. 28).

Ciò richiede al docente di diventare designer (De Rossi \& Ferranti, 2017 p. 12); il design è un'operazione euristica e creativa la quale, pur non sottraendosi a regole di fondo, sottende l'apertura al "dialogo tra le idee e il mondo, tra la teoria e la sua applicazione, tra un concetto e la sua realizzazione, tra gli strumenti e gli obiettivi" (Mishra \& Koheler, 2003, p. 106).

In tale prospettiva l'adozione di procedure di progettazione integrata, secondo il framework ICT-TPCK (Technological, Pedagogical, Content Knowledge) (Angeli \& Valanides 2013; De Rossi \& Trevisan, 2018), può favorire un ragionamento progettuale in grado di armonizzare tutti gli elementi convergenti nella didattica HIS affinché si possano superare discontinuità metodologicotecnologiche, concettuali, procedurali e spazio-temporali. Ossia, accanto agli elementi classici (contenuti, obiettivi, strategie, valutazione) è importante che siano assunte anche le specifiche componenti principali, spesso implicite, del costrutto "approccio didattico" (scelta critica di modelli, metodi, format, tecniche) pensate in accordo con: la scelta consapevole delle tecnologie; le attività di apprendimento con le tecnologie e le forme di conoscenza che esse sollecitano (Harris \& Hofer, 2009); le molteplici modalità di rappresentazione di significato consentite dalle tecnologie (Cope \& Kalantzis, 2000). Questo sembra consentire la costruzione di un'azione didattica coerente, comprensiva del potenziale offerto dalle ICT, anche verso la valorizzazione di soft skill (La Marca \& Gülbay, 2018). Pur essendo un campo d'indagine molto sviluppato, per quanto riguarda la domanda principale della didattica come?, rimangono ancora aperte piste di lavoro che inducono a focalizzare lo sguardo verso l'innovazione di procedure, strategie e strumenti in azione.

\section{II disegno di ricerca: il gruppo di riferimento, metodologia, fasi, strumenti}

Lo studio empirico è stato svolto nell'a.a. 2017-18 con l'obiettivo di stimolare i docenti a progettare soluzione metodologiche-tecnologiche in grado di valorizzare soft skill specifiche nell'ambito di insegnamenti disciplinari curricolari; l'ipotesi è che la progettazione didattica integrata, assumendo la tecnologia come "partner cognitivo" in grado di amplificare l'efficacia del repertorio metodologico, possa attivare processi d'insegnamento-apprendimento per lo sviluppo di competenze trasversali (soft skill) contestualizzate in ambiente tecnologico: creatività, comunicazione efficace, collaborazione (Fredrickson, 2003; Zhou \& George, 2003; Boyatzis, Good \& Massa, 2012). Sono stati coinvolti 42 docenti volontari di un'università italiana $(\mathrm{N} 21)$ e di una straniera nord euro- 
pea (N 21) in cui è diffuso l'uso della piattaforma Moodle (età media 43,99 anni; M. 56\%, F. 44\%; distribuzione 38\% Human Science, 27\% Life Science, 35\% Hard Science). Sono stati considerati sia docenti a contratto $(26,9 \%)$, sia strutturati $(73,1 \%)$ e tutti avevano maturato almeno 3 anni di esperienza didattica universitaria. La maggioranza aveva avuto almeno un'occasione di formazione all'uso di Moodle e di risorse tecnologiche per la didattica (università italiana $38,09 \%$; università straniera 76,19\%). Il 63,5\% ha dichiarato di utilizzare abbastanza e molto le ICT nella didattica (liv. 3 e 4 ), il restante $36,5 \%$ poco e nessuno niente.

Partendo dal presupposto di mettere in campo una procedura ICT-TPCK attraverso modalità $H I S$, convergenti in format blended learning-BL $(30 \%)$, i docenti sono stati invitati a ri-pensare all'azione didattica ponendo come obiettivo lo sviluppo delle soft skill. Il laboratorio di progettazione è stato svolto secondo il format Briefing for Design (BD) (De Rossi, Ferranti, 2017), scegliendo uno o più obiettivi del proprio syllabus da sviluppare in modalità $B L$ impiegando specifiche risorse tecnologiche. Le soluzioni miste a cui si è dato spazio nella scelta sono state flessibili in modo da dare l'opportunità di mescolare onsite learning e online learning (apprendimento collaborativo secondo i canoni online education) (Graham, Woodfield \& Harrison, 2013; Trentin 2015). La fase I ha compreso diverse azioni propedeutiche allo svolgimento delle attività didattiche vere e proprie: la formazione di base metodologico-tecnologica su risorse avanzate per la didattica, interne ed esterne alla piattaforma (ad es. Kaltura; Mobile Storytelling Platform); la realizzazione dei laboratori progettuali $B D$, individuali o in piccolo gruppo per docenti afferenti alla stessa area scientifica.

La realizzazione dei $B D$ ha previsto l'azione congiunta dei docenti, di un docente di didattica generale e di un learning technologist ${ }^{2}$ (Khosrow-Pour, 2014) per la costruzione di format $H S$-BL, active learning per attivare le soft skill considerate, selezionando risorse tecnologiche con affordance ${ }^{3}$ adeguate (Fig. 1).

\footnotetext{
${ }^{2}$ Figura di supporto progettuale-organizzativa con competenze di tecnologie per la didattica; profilo specifico per Teaching Learning Center o Teaching Learning Service.

${ }^{3}$ Il concetto di "affordance", sviluppato da Gibson negli anni '50 e ripreso da Norman (1998), indica le caratteristiche percepibili di oggetti e ambiente che non vengono percepiti in sé, come sono, come pure forme, ma anche per come possono servire a scopi diversi. Un'affordance è un'opportunità d'uso, d'azione o inibizione (Bonaiuti, 2005). In didattica la riflessione in tali termini per le ICT rappresenta un aspetto fondamentale nella progettazione.
} 
Education Sciences \& Society, 2/2018

Fig. 1 - Distribuzione delle attività e delle risorse tecnologiche progettate dai docenti

\begin{tabular}{|c|l|c|}
\hline \multicolumn{2}{|c|}{ Activity/Technological resources } & $\begin{array}{c}\% \\
\text { Teachers }\end{array}$ \\
\hline \multirow{2}{*}{} & Video and Multimedia production (for example Kaltura) & $28 \%$ \\
\cline { 2 - 3 } & $\begin{array}{l}\text { Digital Storytelling production (use of external resources, for example } \\
\text { Mobile Visual Storytelling) }\end{array}$ & $26 \%$ \\
\cline { 2 - 3 } & Group participation in gamification activities & $8 \%$ \\
\cline { 2 - 3 } & Case analysis group activities & $27 \%$ \\
\cline { 2 - 3 } & Group activity of professional problem solving & $24 \%$ \\
\cline { 2 - 3 } & Group activities for the construction of glossaries & $2 \%$ \\
\cline { 2 - 3 } & $\begin{array}{l}\text { Group activities for the construction of artifacts (for example resource Book } \\
\text { of Moodle) }\end{array}$ & $22 \%$ \\
\hline \multirow{2}{*}{ के } & & \\
\hline
\end{tabular}

Per comodità abbiamo raggruppato le risorse in 2 macro-categorie di funzione: i) produzione di artefatti (a, b, f, g); ii) attività di analisi e soluzione di problemi (c, d, e). Come si può notare la maggior distribuzione di evidenzia nell'opzione per attività in gruppo di produzione di artefatti (Video and Multimedia; Digital Storytelling; elaborazione di materiali con risorsa Libro); seguono le attività di Case Analysis e Problem solving, anche in ottica professionalizzante, concentrate maggiormente in insegnamenti di CdS magistrali; bassa è stata la scelta di attività di gamification per la complessità di realizzazione da parte dei docenti (dato estratto dai self-report) e per la costruzione di glossari, scelti in insegnamenti di aree specifiche (es. linguistica). Alcune attività sono state oggetto di scelta combinata in format complessi (ad esempio analisi caso-problem solving abbinati; costruzione di artefatti con risorsa Libro integrando video). La fase II del percorso di ricerca ha riguardato lo svolgimento delle azioni didattiche e il loro monitoraggio (circa 3 mesi). L'approccio mixed-method (Teddlie $\&$ Tashakkori, 2009) ha consentito l'uso di differenti strumenti rivolti sia ai docenti che agli studenti: questionario strutturato somministrato in forma anonima online, in entrata e in uscita ( 4 dimensioni: 18 item, scala Likert a 4 livelli di accordo, $1=$ per niente; $2=$ poco; $3=$ d'accordo; $3=$ molto d'accordo), su cui sono state effettuate 2 revisioni pilot test. È stato indirizzato a rilevare le percezioni sulle seguenti dimensioni: a) procedura di progettazione integrata (4 item); b) complementarità affordance tecnologica delle risorse utilizzate e approccio metodologico (5 item); c) integrazione delle ICT per sviluppo delle soft skill (5 item); organizzazione didattica (4 item). È stato anche utilizzato un ulteriore strumento di tipo qualitativo (self report) al fine di stimolare processi riflessivi per tutta la durata dell'esperienza. In questo articolo, per economia di sintesi, verranno presentati solo i principali dati relativi al questionario somministrato ai docenti. 


\section{I risultati del questionario: le percezioni dei docenti in entrata e in uscita}

Di seguito verranno presentati i dati emersi in entrata e in uscita dagli item relativi alle quattro dimensioni del questionario strutturato (originariamente in lingua inglese, per il presente articolo tradotto in lingua italiana). La somministrazione in entrata è stata effettuata all'inizio dei laboratori di progettazione $B D$ e in uscita al termine dei corsi.

I rispondenti in entrata e in uscita sono stati la totalità (N 42). Si propone la riflessione sui risultati riportandoli in tabelle distinte per dimensioni; per ogni item è stata effettuata la media $(\mathrm{M})$ dei livelli di risposta in entrata e in uscita. Sono stati considerati come valori neutri quelli assestati su $\mathrm{M}=2.5$; sono stati considerati con trend positivo $\mathrm{M}>2.5$, (livello 3-d'accordo; livello 4-molto d'accordo), mentre con trend negativo i valori $\mathrm{M}<2.5$; (livello 1-per niente d'accordo; livello 2-poco d'accordo).

\section{Progettazione integrata}

La dimensione a) "Progettazione integrata" (4 item), ha inteso indagare la percezione dei docenti sul focus specifico "progettazione" tenendo conto che è un'azione necessaria per la predisposizione dei syllabus. Si è verificato che in entrambe le Istituzioni di appartenenza non erano mai state attivate formazioni specifiche, né erano presenti risorse di supporto finalizzate e quindi le percezioni in entrata sono state considerate riferibili alle esperienze didattiche spontaneamente maturate. Per quanto riguarda il riferimento al framework ICT-TPCK, implicitamente sotteso agli item 1 e 3 (Fig. 2) si notano evidenze di cambiamento di percezione con trend inverso: nell'I.1 il livello medio riscontrato in uscita si abbassa, mentre nell'I.3 aumenta. Ossia, nell'I.1 in uscita, dopo aver progettato e lavorato con gli studenti in modo innovativo, i docenti sembrano aver percepito in modo marcato che per insegnare efficacemente non è sufficiente solo la conoscenza dei contenuti della propria disciplina. Ciò può essere collegato all'andamento dei dati dell'I.3, la cui media inizialmente evidenziava come fosse poco percepita l'importanza di saper coniugare contenuti, metodologie e tecnologie nella progettazione; percezione ampiamente modificata, invece, in uscita dell'esperienza $H S-B L$ attraverso cui evidentemente i docenti hanno avuto modo di ampliare lo sguardo verso la complessità degli elementi in azione nella didattica, oltre i soli contenuti. 
Fig. 2 - Item dimensione "Progettazione integrata", $M$ in entrata e in uscita

\begin{tabular}{|l|c|c|}
\hline Dimensione A-Progettazione Integrata & $\begin{array}{c}\text { M } \\
\text { entrata }\end{array}$ & $\begin{array}{c}\text { M } \\
\text { uscita }\end{array}$ \\
\hline $\begin{array}{l}\text { I.1) Nella progettazione didattica è prevalente la conoscenza dei conte- } \\
\text { nuti disciplinari }\end{array}$ & 3,64 & 1,83 \\
\hline $\begin{array}{l}\text { I.2) Le ICT aiutano i docenti a trasporre i contenuti in prospettiva di } \\
\text { competenza }\end{array}$ & 2,44 & 3,02 \\
\hline $\begin{array}{l}\text { I.3) II docente deve essere in grado di progettare il percorso didattico } \\
\text { coniugando appropriatamente i contenuti disciplinari di insegnamento, } \\
\text { le metodologie didattiche e le ICT adeguate }\end{array}$ & 2,37 & 3,25 \\
\hline $\begin{array}{l}\text { I.4) Progettare integrando le ICT consente di monitorare i processi di } \\
\text { apprendimento }\end{array}$ & 2,65 & 3,47 \\
\hline
\end{tabular}

Interessante è anche il dato sul cambiamento della percezione di utilità delle ICT nei processi d'insegnamento (I.2-trasposizione in prospettiva di competenza; I.4-valutazione dei processi). Dopo l'esperienza, infatti, sembrano aver maggiormente percepito la potenzialità data dall'integrazione delle ICT sia in riferimento al superamento della didattica trasmissiva, verso quella per competenze, sia in relazione alla possibilità di attuare valutazione di processo, difficile da realizzare con un approccio tradizionale.

\section{Complementarità tra affordance tecnologica delle risorse utilizzate e approccio metodo- logico}

La seconda dimensione b) "Complementarità tra affordance tecnologica delle risorse utilizzate e approccio metodologico" (5 item) ha focalizzato con maggiore approfondimento la questione critica della conoscenza delle risorse tecnologiche declinata didatticamente, motivo per cui la letteratura sulla competenza digitale (ad es. Ala Mutka, 2011) ha sottolineato che la conoscenza in sé degli strumenti è solo pre-requisito per i docenti, ma non è sufficiente per un'adozione delle ICT in grado di supportare effettivamente l'innovazione didattica. Il concetto di affordance, così come quelli di forma di conoscenza e rappresentazione della conoscenza sono stati ampiamente discussi durante la fase di formazione in modo da poter consentire consapevolezza nelle risposte. Gli item (Fig. 3) hanno stimolato una riflessione generale sul rapporto tra affordance e approccio metodologico, considerando che in qualsiasi format didattico vengono utilizzate diversificate risorse. Per approccio metodologico si intende la scelta nell'insieme di format, strategie, tecniche e strumenti che caratterizzano l'azione didattica sul campo. 
Fig. 3 - Dimensione "Complementarità tra affordance tecnologica delle risorse utilizzate e approccio metodologico", M in entrata e in uscita

\begin{tabular}{|l|c|c|}
\hline $\begin{array}{l}\text { Dimensione B-Complementarità tra affordance tecnologica delle } \\
\text { risorse utilizzate e approccio metodologico }\end{array}$ & $\begin{array}{c}\text { M } \\
\text { entrata }\end{array}$ & $\begin{array}{c}\text { M } \\
\text { uscita }\end{array}$ \\
\hline $\begin{array}{l}\text { I.1) Per sviluppare un'azione didattica efficace è importante riflettere } \\
\text { sulle affordance specifiche di ogni risorsa tecnologica }\end{array}$ & 2,12 & 3,64 \\
\hline $\begin{array}{l}\text { I.2) Le risorse tecnologiche (ICT) consentono di diversificare le forme di } \\
\text { conoscenza nella didattica }\end{array}$ & 2,85 & 3,03 \\
\hline $\begin{array}{l}\text { I.3) Le risorse tecnologiche (ICT) consentono di diversificare le forme di } \\
\text { rappresentazione della conoscenza nella didattica }\end{array}$ & 2,74 & 3,25 \\
\hline $\begin{array}{l}\text { l.4) L'integrazione delle ICT nella didattica facilitano approcci Active } \\
\text { Learning }\end{array}$ & 2,35 & 3,56 \\
\hline $\begin{array}{l}\text { I.5) L'affordance delle risorse tecnologiche è complementare alla scelta } \\
\text { dell'approccio metodologico }\end{array}$ & 2,06 & 2,97 \\
\hline
\end{tabular}

Generalmente si può notare che in entrata tutti gli item riportano una media inferiore al livello 3 , anche se I.2 e I.3 sono situati con trend lievemente positivo $\mathrm{M}>2.5$, mentre in uscita lo spostamento è orientato verso livelli a trend positivo (nel caso di I.1 e I.4, M>3,5). Nello specifico, quanto riguarda la questione del rapporto percepito tra affordance delle risorse tecnologiche e scelta dell'approccio metodologico, in entrambi gli item esplicitamente dedicati (I.1 e I.5), si nota in uscita un aumento di livello di accordo, così come la percezione di potenzialità derivata dall'integrazione delle ICT nella didattica per l'adozione di un approccio active learning (I.4).

Quindi la formazione, l'accompagnamento progettuale e l'esperienza riflessiva sul campo previsti dal progetto di ricerca, sembrano aver sortito un interessante impatto sui docenti partecipanti, quanto meno in termini di percezione. Infatti, complessivamente, le modificazioni registrate in uscita sembrano accordarsi con la prospettiva emersa da altri studi in letteratura secondo cui l'insegnante, di qualsiasi tipo, grazie all'adozione di procedure progettuali integrate ICT-TPCK è stimolato ad agire più come "designer", superando il ruolo "consumer" di modelli pre-costruiti (Niess, 2015).

Integrazione delle ICT per sviluppo delle soft skill in ambiente tecnologico (creatività, comunicazione, collaborazione)

La terza dimensione $(5$ item) riguardante le percezioni sulla potenzialità di sviluppo di soft skill derivate dell'uso integrato delle tecnologie nella didattica, 
ha aperto a interessanti riflessioni date dagli esiti (Fig. 4). In particolare l'I.3 ha inteso cogliere le percezioni dei docenti relativamente alla possibilità di considerare l'approccio soft-skilloriented in insegnamenti curricolari non specificatamente dedicati (ad es. lo sviluppo di comunicazione efficace può essere un obiettivo trasversale da porre e valutare anche in insegnamenti che non siano centrati sui contenuti della comunicazione e così per la creatività o per la collaborazione). Infatti, da oltre vent'anni, nella formazione universitaria è ormai assodata l'importanza dello sviluppo di competenze trasversali, tuttavia la complessità della connessione tra hard e soft skill - da progettare, attuare con una didattica mirata e valutare - sembra ancora questione complessa da realizzare pienamente.

Fig. 4 - Dimensione "L'integrazione delle ICT per sviluppo delle soft skill in ambiente tecnologico (creatività, comunicazione, collaborazione)", $M$ in entrata e in uscita

\begin{tabular}{|l|c|c|}
\hline $\begin{array}{l}\text { Dimensione C-L'integrazione delle ICT per sviluppo delle soft } \\
\text { skill in ambiente tecnologico (creatività, comunicazione, colla- } \\
\text { borazione) }\end{array}$ & $\begin{array}{c}\text { M } \\
\text { entrata }\end{array}$ & $\begin{array}{c}\text { M } \\
\text { uscita }\end{array}$ \\
\hline $\begin{array}{l}\text { I.1) L'integrazione delle ICT nella didattica curricolare consente l'attiva- } \\
\text { zione di processi creativi }\end{array}$ & 2,03 & 2,89 \\
\hline $\begin{array}{l}\text { I.2) L'integrazione delle ICT nella didattica curricolare consente l'attiva- } \\
\text { zione di processi comunicativi }\end{array}$ & 2,85 & 3,43 \\
\hline $\begin{array}{l}\text { I.3) È possibile sviluppare progettazioni didattiche curricolari che met- } \\
\text { tano in connessione obiettivi di competenza disciplinare (hard skill) e } \\
\text { obiettivi di competenza trasversale (soft) }\end{array}$ & 1,89 & 3,05 \\
\hline $\begin{array}{l}\text { I.4) Ambienti d'apprendimento integrato con ICT consentono lo sviluppo } \\
\text { di processi collaborativi nell'apprendimento. }\end{array}$ & 2,37 & 3,55 \\
\hline $\begin{array}{l}\text { I.5) Ambienti d'apprendimento integrato con ICT consentono la valuta- } \\
\text { zione di soft skill }\end{array}$ & 1,95 & 2,94 \\
\hline
\end{tabular}

In entrata la relazione tra uso di ICT nella didattica e sviluppo di processi comunicativi (I. 2, M=2,85) è sembrata essere quella maggiormente percepita dai docenti, aumentando ampiamente la tendenza di livello in uscita, dopo l'esperienza sul campo $(\mathrm{M}=3,43)$. Per quanto riguarda le altre soft skill specificatamente considerate (I.1 e I.4), valutate a livello $\mathrm{M}<2.5$ in entrata, si evidenzia un aumento con trend positivo in uscita, soprattutto per quanto riguarda i processi collaborativi. I dati più interessanti in fase di analisi sono derivati dagli I. 3 e I. 5, riferiti il primo alla già citata questione della progettazione curricolare soft-skilloriented e il secondo alla valutazione; infatti, entrambi gli item in entrata presentavano trend decisamente negativi $(M<2)$ che in uscita si sono inve- 
ce attestati intorno a un livello positivo, $\mathrm{M}$ in zona 3. Ciò consente di ipotizzare che procedure specifiche di formazione dei docenti, supporto nella progettazione e implementazione di modalità $H S$ consentano nell'azione didattica curricolare una maggior centratura su obiettivi formativi complessi, comprendenti anche lo sviluppo e la valutazione di competenze trasversali.

\section{Organizzazione didattica}

L'ultima dimensione del questionario, 4 item (Fig. 5), ha inteso descrivere il quadro complessivo di alcune questioni ritenute dal gruppo di ricerca maggiormente rilevanti perché più diffusamente riscontrate in letteratura (Picciano, Dziuban, \& Graham, 2014; Pratt \& Kovatcheva, 2018).

Fig. 5 - Dimensione "Organizzazione didattica HS-BL", $M$ in entrata e in uscita

\begin{tabular}{|l|c|c|}
\hline Dimensione D-Organizzazione didattica HS-BL & $\begin{array}{c}\text { M } \\
\text { entrata }\end{array}$ & $\begin{array}{c}\text { M } \\
\text { uscita }\end{array}$ \\
\hline $\begin{array}{l}\text { l.1) La didattica HS-BL è utile per risolvere problemi e/o criticità orga- } \\
\text { nizzative (gestione dei tempi; gestione dello spazio) }\end{array}$ & 3,64 & 3,87 \\
\hline $\begin{array}{l}\text { l.2) In caso non sia obbligatoria la frequenza in presenza ma lo sia } \\
\text { nelle attività a distanza, ritrovare gli studenti nella comunità virtuale } \\
\text { può stimolare la loro partecipazione attiva ai processi di insegnamento/ } \\
\text { apprendimento }\end{array}$ & 2,44 & 3,52 \\
\hline $\begin{array}{l}\text { l.3) Allestire un ambiente d'apprendimento in modalità HS-BL è utile } \\
\text { per dare supporto metodologico agli studenti nei processi d'apprendi- } \\
\text { mento }\end{array}$ & 2,37 & 3,25 \\
\hline $\begin{array}{l}\text { l.4) Avere la possibilità di progettare le attività didattiche con il suppor- } \\
\text { to di un gruppo di esperti metodologici e tecnologici aiuta a innovare la } \\
\text { propria didattica }\end{array}$ & 2,65 & 3,85 \\
\hline
\end{tabular}

Complessivamente le medie dei livelli in uscita di tutti i 4 item sono risultate ampiamente positive $M>3$. In particolare, l'I.1 si avvicina al livello più elevato di accordo in riferimento alla percezione di utilità della didattica HS-BL per risolvere criticità organizzative, come la gestione del tempo o degli spazi, consentendo maggiore flessibilità e soluzioni diversificate grazie alla commistione onsite/online learning. Apprezzato è stato anche il supporto di esperti che sembra aver stimolato maggiormente i docenti al cambiamento delle proprie routine didattiche, verso l'assunzione di una prospettiva innovativa. Gli item, I.2 e I.3 hanno sottolineato una positiva percezione di utilità della di- 
dattica $H S-B L$ in merito alla maggior partecipazione attiva riscontrata negli studenti e alla possibilità di offrire supporto nell'apprendimento.

\section{Conclusioni}

Seppur i dati presentati siano solo una parte di quanto indagato nella ricerca, consentono alcune sintetiche considerazioni utili a porre l'attenzione su questioni non completamente definite nella prassi didattica universitaria e sulle quali la ricerca sta continuando a impegnarsi. Riprendendo anche Trentin (2015, p. 28) è possibile concludere che "affinché le HIS possano fungere da leva per favorire pratiche didattiche innovative e livelli di apprendimento significativi, dovrebbero essere progettate in modo da supportare strategie collaborative, centrate sul protagonismo degli studenti e, al contempo avere al loro interno strumenti funzionali alla valutazione degli apprendimenti”. La progettazione di ambienti d'apprendimento integrati sembra rendere attuabile una didattica softskilloriented sia grazie a metodi e strumenti considerati alla luce di tutti gli elementi della progettazione ICT-TPCK, sia se basata su scelte pedagogiche riguardo la necessaria complementarietà e il dosaggio delle componenti onsite/ online del processo di insegnamento-apprendimento (McGee, 2014).

\section{Riferimenti bibliografici}

Ala-Mutka K. (2011). Mapping Digital Competence: Towards a Conceptual Understanding. Luxembourg: Publications Office of the European Union. http://ftp.jrc.es/EURdoc/JRC67075_TN.pdf

Angeli C., \& Valanides N. (2009). Epistemological and methodological issues for the conceptualization, development, and assessment of ICT-TPCK: Advances in Technological Pedagogical Content Knowledge (TPCK). Computers \& Education, 52(1): 154-168. doi:10.1016/j.compedu.2008.07.006

Angeli C., \& Valanides N. (2013). Technology mapping: An approach for developing technological pedagogical content knowledge. Journal of Educational Computing Research, 48(2): 199-221. https://doi.org/10.2190\%2FEC.48.2.e

Bonaiuti G. (2005). Strumenti della rete e processo formativo. Firenze: Firenze University Press

Boyatzis R.E., Good D., \& Massa R. (2012). Emotional, social, and cognitive intelligence and personality as predictor of sales leadership performance. Journal of Leadership \& Organizational Studies, 19(2): 191-201. https:// journals.sagepub.com/doi/abs/10.1177/1548051811435793

Cope B., \& Kalantzis M. (Eds.) (2000). Multiliteracies: Literacy learning and the design of social futures. London: Routledge. 
De Rossi M., \& Ferranti C. (2017). Learning by design dell'intersezione tra discipline, metodologie didattiche e tecnologie. Italian Journal of Educational Research, 10, 241-253.

De Rossi M., \& Trevisan, O. (2018). Technological Pedagogical Content Knowledge in the literature: how TPCK is defined and implemented in initial teacher education. Special Issue, Italian Journal of Educational Technology, vol. 26, 1.

Dziuban C., Moskal P., Kramer L., \& Thompson J. (2013). Student satisfaction with online learning in the presence of ambivalence: Looking for the will-o'the-wisp. The Internet and Higher Education, 17: 1-8. http://dx.doi.org/10.1016/j. iheduc.2012.08.001

Khosrow-Pour M. (2014). Educational Technology Use and Design for Improved Learning Opportunities. Heshey: Information Science Reference-IGI Global.

Fredrickson B.L. (2003). Positive emotions and upward spirals in organizations. In: K.S. Cameron, J.E. Dutton \& R.E. Quinn (Eds.), Positive organizational scholarship: foundations of a new discipline. San Francisco, CA: Berrett-Koehler Publishers.

Graham C.R., Woodfield W., \& Harrison, J.B. (2013). A framework for institutional adoption and implementation of blended learning in higher education. Internet and Higher Education, 18(3): 4-14. https://doi.org/10.1016/j.iheduc.2012.09.003

Harris J., \& Hofer M. (2009). Instructional planning activity types as vehicles for curriculum-based TPACK development. In: C.D. Maddux (Ed.), Research highlights in technology and teacher education 2009. Chesapeake, VA: Society for Information Technology in Teacher Education (SITE).

Kaleta R., Skibba K., \& Joosten T. (2007). Discovering, designing, and delivering hybrid courses. In: A.G. Picciano \& C.D. Dziuban (Eds.), Blended Learning: Research Perspectives. Needham, MA: Sloan Consortium.

La Marca A., Gülbay E. (2018). Didattica universitaria e sviluppo delle soft skill. Lecce: Pensa Multi Media.

McGee P. (2014). Blended course design: Where's the pedagogy? International Journal of Mobile and Blended Learning, 6(1), 33-55. https://doi.org/10.4018/ ijmbl.2014010103

Mishra P., \& Koehler M.J. (2003), Not "What" but "How": Becoming Design-Wise about Educational Technology. In: Y. Zhao (Ed.), What should teacher know about technology: Perspectives and practices. Greenwich, CT: Information Age Publishing.

Niess M.L. (2015). Transforming teachers' knowledge: Learning trajectories for advancing teacher education for teaching with technology. In C. Angeli \& N. Valanides, Technological pedagogical content knowledge. Exploring, developing, and assessing TPCK. NY: Springer.

Norman D.A. (1998). The Invisible Computer. Cambridge: MITPress.

Picciano A.G., Dziuban C.D., \& Graham C.R. (2014). Blended learning: Research perspectives, vol. 2. New York, NY: Routledge.

Pratt K., \& Kovatcheva E.P. (2018). Designing Blended, Flexible, and Personalized Learning. In: J. Voogt, G. Knezek, R. Christensen, K.W. Lai (Eds.), Second Handbook of Information Technology in Primary and Secondary Education. Chambridge: Springer International Handbooks of Education. 
Shea P. (2007). Towards A Conceptual Framework for Learning in Blended Environments. In: A. Picciano \& C. Dziuban (Eds.), Blended Learning. Research Perspectives. Needham, MA: Sloan Consortium

Teddlie C., \& Tashakkori A. (2009). Foundations of mixed methods research: Integrating quantitative and qualitative approaches in the social and behavioral sciences. Thousand Oaks, CA: Sage.

Trentin G., \& Bocconi S. (2015). Didattica ibrida e insegnamento universitario: linee guida per una progettazione efficace. Italian Journal of Educational Research, VIII: 27-42. ISSN 2038-9744

Zhou J., \& George J.M. (2003). Awakening employee creativity: the role of leader emotional intelligence. The Leadership Quarterly, 14: 545-569. doi:10.1016/S10489843(03)00051-1 